\title{
Design, development and performance characteristics of a large aperture disc amplifier for high power Nd: Glass laser chain
}

\author{
M P KAMATH, P K TRIPATHI, A P KULKARNI, R CHANDRA, \\ A S JOSHI, C P NAVATHE and P D GUPTA
}

Laser Plasma Division, Raja Ramanna Centre for Advanced Technology, Indore 452013

e-mail: kamath@cat.ernet.in

MS received 26 July 2007; revised 15 November 2007

\begin{abstract}
A large aperture disc amplifier has been designed, set-up and characterized for its performance on small signal gain, spatial variation of gain, and thermal recovery time. This amplifier, consisting of three elliptical Nd: phosphate glass discs of size $214 \times 114 \times 20 \mathrm{~mm}$ mounted at Brewster angle and pumped by ten xenon filled flash lamps of $600 \mathrm{~mm}$ arc length, provided a small signal gain of 6 at electrical pump energy of $36 \mathrm{~kJ}$ (in a pulse of $450 \mu \mathrm{s}$ ) using an in-house developed dual-polarity capacitor bank based power supply. It was coupled to a high power $\mathrm{Nd}$ : phosphate glass laser chain and a maximum output pulse energy exceeding $100 \mathrm{~J}$ in a $1.5 \mathrm{~ns}$ (FWHM) pulse has been measured. A dry nitrogen gas based cooling system was developed for cooling the glass discs with a thermal recovery time of $\sim 20$ minutes.
\end{abstract}

Keywords. Disc amplifier; high power lasers; dual polarity power supply; dry nitrogen cooling system.

\section{Introduction}

High energy high power (HEHP) laser systems based on master oscillator power amplifier (MOPA) architecture (Campbell et al 1986, Boehly et al 1987, Joshi et al 1999) are widely used as pulse power drivers for studies of inertial confinement fusion, generation of intense thermal x-ray radiation, pumping of x-ray lasers, etc. In MOPA-based laser systems, the laser pulse from the master oscillator is amplified in successive laser amplifiers of increasing beam cross-sections to keep the laser intensity below the damage threshold of the optical components. A majority of the HEHP laser chains use Nd: phosphate glass as the active medium because of its smaller coefficient of intensity-dependent nonlinear refractive index and higher stimulated emission cross-section (Campbell \& Suratwala 2000). These systems are multi-arm lasers seeded with pulses of duration ranging from a few $100 \mathrm{ps}$ to few ns from the master oscillator and deliver laser pulse energy ranging from $100 \mathrm{~J}$ to few $\mathrm{kJ}$ from each arm. For laser pulse energy of $1 \mathrm{~kJ}$, the beam diameter of the order of several centimeters is 
required to operate laser amplifiers below laser induced damage threshold (LIDT) of optics, which typically lies in the range of $2-3 \mathrm{GW} / \mathrm{cm}^{2}$ for $1 \mathrm{~ns}$ laser pulse. These lasers use xenon flash lamps as the pump sources for their amplifiers. This puts restrictions on operation of high power $\mathrm{Nd}$ glass lasers to single shot mode due to thermal management problems. The latter will be overcome in future with the development of large laser diode arrays.

The large diameter rod amplifiers of HEHP laser chains, suffer from the problem of spatial gain variation, thermal lensing and stress-induced birefringence because they are optically pumped and cooled through the cylindrical face. The problem of non-uniformity gain may be reduced with proper choice of rod diameter and $\mathrm{Nd}$ doping concentration by satisfying the relation $\rho \mathrm{D} \sim 3$, where $\rho$ is the doping density by weight percentage and $\mathrm{D}$ is diameter of laser rod in $\mathrm{cm}$ (Koechner 1988). However, this results in rather poor gain for the large diameter rod amplifiers because of the small doping concentration. In contrast, laser discs with large apertures and small thickness, are optically pumped and cooled through their faces (Boehly et al 1987, Zhang et al 1998, Tou'ze et al 1998). Hence, they have smaller spatial variation of gain and have larger doping densities. Further, since the disc amplifiers use thin discs and are operated with large gain coefficients, they have much smaller non-linear cumulative phase i.e. B integral (Hunt et al 1978). Another advantage is that the laser discs have higher fracture limits and can be operated at higher thermal loads compared to the laser rods.

A two-arm Nd: phosphate glass laser system based on MOPA architecture was developed at Laser Plasma Division, RRCAT, Indore (Joshi et al 1999) delivering laser energy of $50 \mathrm{~J}$ over the pulse duration of $\sim 1.5 \mathrm{~ns}$ in each arm. Both the arms of above laser system are seeded with laser pulses of duration in the range from $600 \mathrm{ps}$ to $1.5 \mathrm{~ns}$ obtained from master oscillator (SL-321 from EXPLA Ltd, Lithuania). The laser has three pre-amplifiers and eight amplifiers. Each of the amplifiers is followed by a spatial filter/optical relay system to control the degradation of spatial profile of the laser by following the concept of optical relaying (Hunt et al 1978). The last rod amplifier stage of the laser chain uses $80 \mathrm{~mm}$ diameter phosphate glass rod. Upgradation of the above laser system requires incorporating disc amplifiers.

In this paper, we report design, development and performance characteristics of a disc amplifier for its use in amplification of $94 \mathrm{~mm}$ diameter laser beam of our HEHP laser system. This amplifier, consists of 3 elliptical Nd: phosphate glass discs of size $214 \times 114 \times 20 \mathrm{~mm}$ mounted at Brewster angle $\left(56.7^{\circ}\right)$ and is pumped by 10 xenon filled flash lamps of $600 \mathrm{~mm}$ arc length. Small signal gain of 6 was obtained at electrical pump energy of $36 \mathrm{~kJ}$ (in $450 \mu \mathrm{s}$ ) using an in-house designed and developed dual polarity capacitor bank based power supply. The output pulse energy in excess of $100 \mathrm{~J}, 1.5 \mathrm{~ns}$ (at a pump energy of $20 \mathrm{~kJ}$ ) has been obtained. The disc amplifier is cooled by dry nitrogen based cooling system providing a thermal recovery time of $\sim 20$ minutes.

\section{Description of disc amplifier and related sub-systems}

\subsection{Opto-mechanical sub-system}

The length of minor and major axes and thickness of discs were 114, 214 and $20 \mathrm{~mm}$ respectively. The $\mathrm{Nd}$ doping concentration in the discs was chosen to be $2.2 \mathrm{wt} \%$ of $\mathrm{Nd}_{2} \mathrm{O}_{3}$ for pump pulse duration of the order of the fluorescence lifetime (of the ${ }^{4} \mathrm{~F}_{3 / 2}$ upper laser level of $\mathrm{Nd}^{3+}$ ion) (Linford et al 1979). The thickness of the discs was decided by considering the absorption of pump light and the loss mechanisms viz. amplified spontaneous emission (ASE) and parasitic oscillations in the discs. While thick discs (typically $\sim 40 \mathrm{~mm}$ ) were 


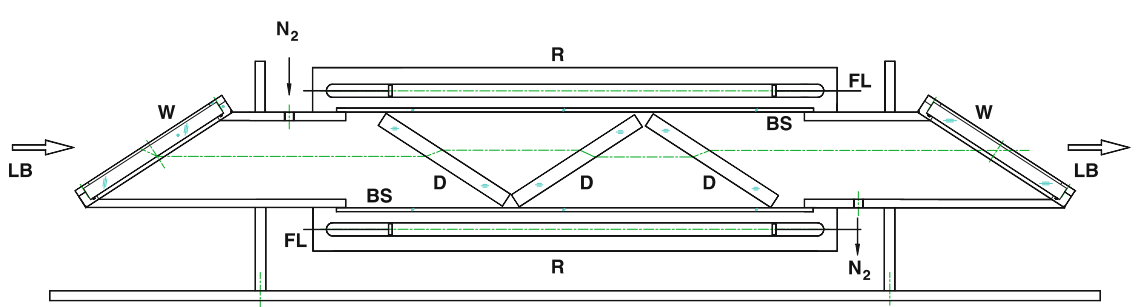

LB-laser beam, FL-flash lamp, D-disc, W- glass window, $\mathrm{N}_{2}$ - nitrogen gas, BS - Blast shield, R-Reflector

Figure 1a. Schematic diagram of the optical scheme of the disc amplifier.

preferred for larger gain, the thinner discs (typically $\sim 15 \mathrm{~mm}$ ) were required to minimize the various losses as described above for the discs of dimensions of $\sim 100 \times 200 \mathrm{~mm}$. The laser discs were also cladded to minimize the parasitic oscillations using a glass material of matching refractive index and high absorption at $1054 \mathrm{~nm}$ (Glaze et al 1974).

Figure 1a shows an optical scheme of the disc amplifier consisting of three Nd: phosphate glass, elliptical shaped discs (LHG-8 from Hoya, Japan) kept at Brewster angle, mounted in aluminum cassettes in a zigzag configuration. The disc amplifiers are usually designed with odd number of discs. Although even number of discs do not produce any net beam line offset due to refraction, odd number of discs have the advantageous feature of canceling asymmetric gain gradients in the two end discs (Wonterghem et al 1997, Erlandson et al 1995). While the metal-based cassettes were chosen for efficient heat conduction, aluminium was chosen in order to decrease the weight of the mechanical housing. A photograph of the disc amplifier assembly is shown in figure $1 \mathrm{~b}$.

Ten xenon flash lamps of $16 \mathrm{~mm}$ bore diameter, $600 \mathrm{~mm}$ arc length and fill pressure of $\sim 400$ torr were used in two sets (of 5 numbers each) and mounted over blast shields of toughened glass. The latter protects the laser discs from damage in the case of explosion of

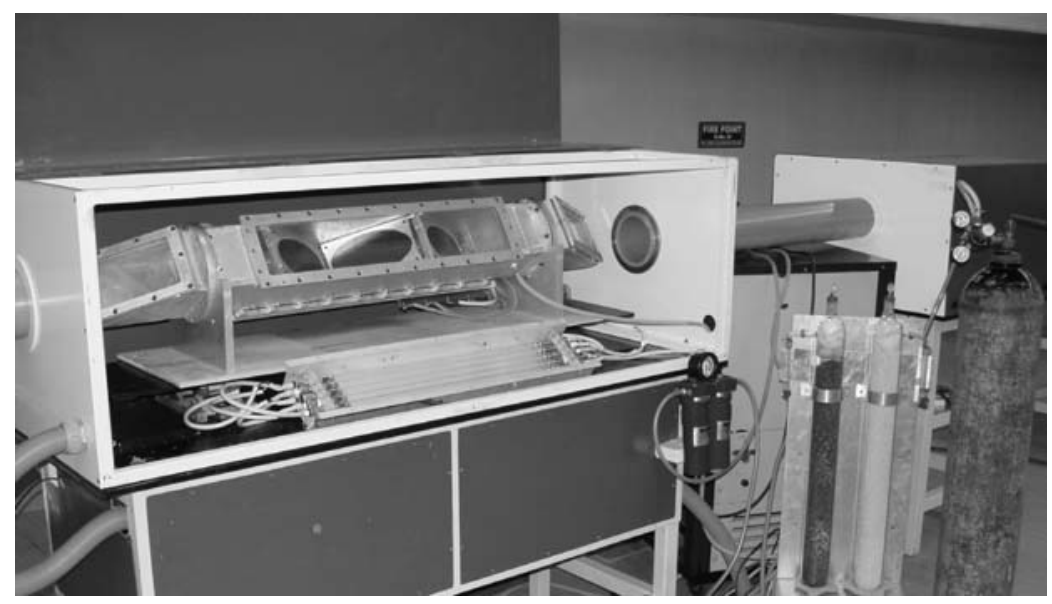

Figure 1b. Photograph of the disc amplifier system. 


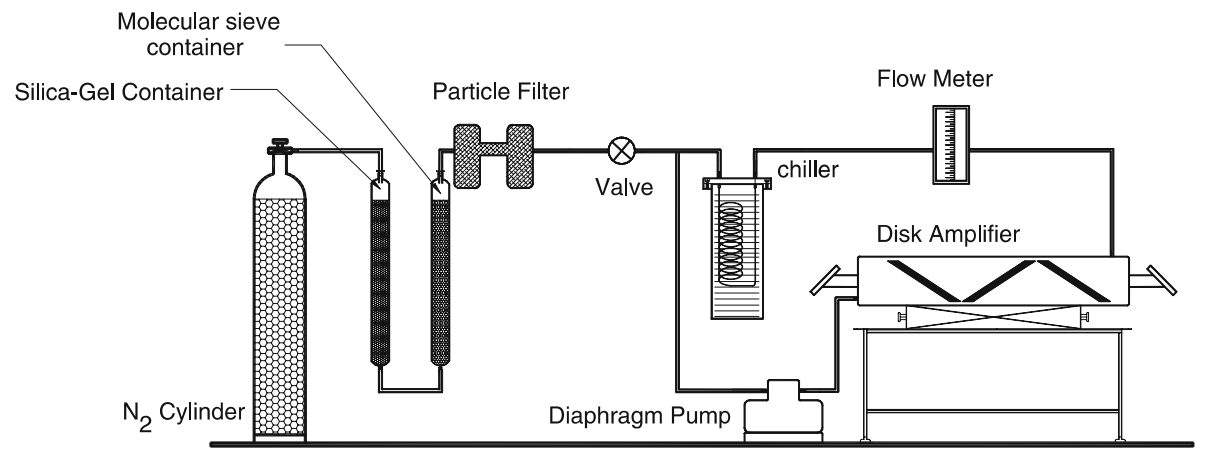

Figure 2. Schematic diagram of the cooling system of the disc amplifier.

the flash lamps. Provision is also made to rotate the disc amplifier assembly around its axis so that linearly polarized laser beam having any polarization axis can be propagated through the disc amplifier without reflection losses. The clear aperture of the disc amplifier was $95 \mathrm{~mm}$, restricted due to refraction of the laser beam.

\subsection{Cooling system}

The total absorbed optical energy in the discs is about $\sim 20 \%$ of the electrical pump energy (Touze et al 1998). This energy increases the temperature of the discs and also leads to undesirable thermal effects. For instance, at electrical pump energy of $36 \mathrm{~kJ}$, the temperature of the glass discs increase by $\Delta T=5^{\circ} \mathrm{C}$. This in turn may lead to a maximum excess optical path of $\sim \delta * \Delta T * l \sim \lambda / 4$, for $l=7.2 \mathrm{~cm}$ and $\delta=0.8 \times 10^{-6} / \mathrm{K}$ (Campbell \& Suratwala 2000). To avoid such phase distortions, cooling of discs is essential. The cooling of discs can be done either directly by flowing dry coolant gas in the disc amplifier housing or indirectly by cooling the flash lamp modules by flowing forced air through them. In the latter case, the heat in the discs is indirectly removed by convection in the amplifier housing followed by the conduction across the blast shields (Sutton et al 1998). This leads to a longer thermal recovery time. Both the cooling methods have their own advantages and disadvantages. For instance, the direct method provides a smaller thermal recovery time (thus possibility of higher repetition rate of laser operation) compared to the indirect method. However, it may cause distortions of the spatial profile due to turbulent flow of the cooling gas. These distortions can be minimized by optimizing the flow rate of the cooling gas and the inlet temperature.

A schematic diagram of the cooling system used for cooling of discs is shown in figure 2 . It uses commercial grade nitrogen gas as the cooling medium. It was observed that the optical quality of the disc surface deteriorates due to the presence of water vapour in commercial grade nitrogen cylinders. Therefore, to avoid the deterioration of optical quality of disc surfaces, a two-stage moisture trap (consisting of $80 \mathrm{~cm}$ silica gel column followed by $80 \mathrm{~cm}$ molecular sieve column) was incorporated in the cooling system. To prevent any dust from entering the disc amplifier, a $0.5 \mu \mathrm{m}$ particle filter was also added in the cooling system. The dry filtered nitrogen gas was cooled in a secondary chiller by circulating it in a copper tube coil that was immersed in a water bath having a temperature of $10^{\circ} \mathrm{C}$. The coolant gas was then circulated in the coil and the disc amplifier module with the help of a diaphragm pump. It was found that the use of moisture trap is effective in maintaining the optical quality of the surface of the disc over long periods of operation (more than 6 months). The performance 


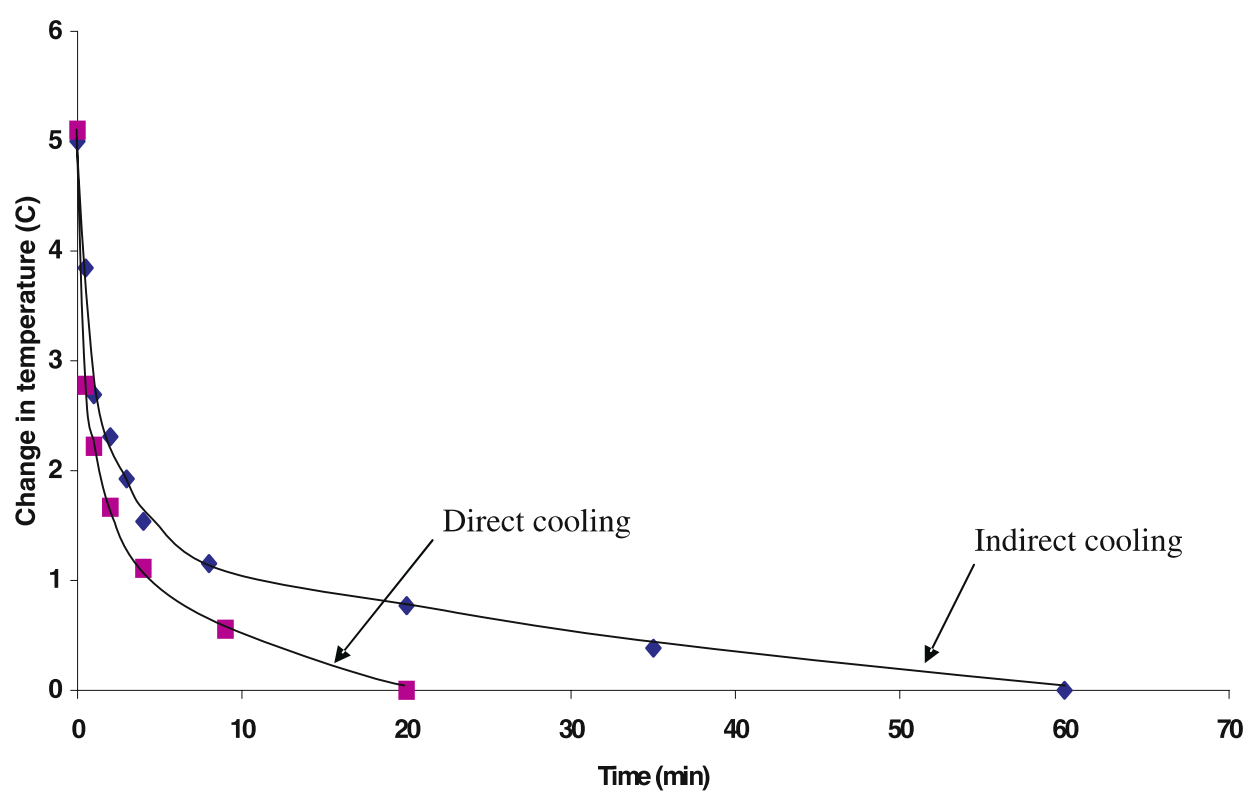

Figure 3. Performance of the cooling system of the disc amplifier.

characteristics vis-à-vis the thermal recovery time for both the direct and indirect methods was studied using a semiconductor temperature sensor (LM35 having temperature sensitivity of $10 \mathrm{mV} /{ }^{\circ} \mathrm{C}$, range of $-55^{\circ} \mathrm{C}$ to $150^{\circ} \mathrm{C}$ ). Figure 3 shows the performance characteristics of the cooling system of the disc amplifier at an input flash lamp pump energy of $36 \mathrm{~kJ}$ using dry nitrogen coolant gas having inlet temperature of $19^{\circ} \mathrm{C}$ and flow rate of $10 \mathrm{lpm}$. A rise in temperature of $\sim 4.4^{\circ} \mathrm{C}$ was observed at the surface of the discs after the flash lamp pump pulse was over. It was found that the discs were cooled to the initial temperature in $\sim 1 \mathrm{hr}$ for indirect and $\sim 20$ minutes for direct cooling method. Next, the phase distortion due to the turbulence of the gas flow and thermo-optical distortions in the discs on laser beam in the case of direct method was also studied using a shear interferometer and was observed to be negligible. Therefore, in our case the direct cooling method was used.

\subsection{Power conditioning and control system}

Two modules each consisting of five flash lamps have been used to pump the discs from either side of the disc amplifier housing. While the number of flash lamps required to pump the disc amplifier was chosen considering the geometrical factor and the explosion energy, the arc length of the flash lamps was chosen in accordance with the effective physical length of three laser discs kept at Brewster angle. In the present case, the flash lamps with arc length of $600 \mathrm{~mm}$ and bore diameter of $16 \mathrm{~mm}$ are operated at 20-30\% of explosion energy (Zhang et al 1998) for a lifetime exceeding 1 million shots. For these flash lamps the explosion energy is $21 \mathrm{~kJ}$ for the case of a critically damped pulse of pulse width $400 \mu \mathrm{s}$ (Bervekk 1975). Thus for delivering an electrical pump energy of $40 \mathrm{~kJ}$, each flash lamp can be operated at $4 \mathrm{~kJ}$ energy, which is $20 \%$ of the explosion energy. The cavity pumping efficiency exponentially decays as a function of the current density in the flash lamp (Linford et al 1979) because of increase in temperature of the flash lamp at high current densities, which eventually makes them more opaque to the reflected pump light. Operational current density of the flash lamps 
is therefore, kept in the range $1-2 \mathrm{kA} / \mathrm{cm}^{2}$ for a pumping efficiency of $\sim 30-40 \%$ (Linford et al 1979). The design of our power supply ensures that the flash lamps are operated with current density varying between 1.2 and $1.7 \mathrm{kA} / \mathrm{cm}^{2}$.

An optimized pulse-forming network (PFN) (of optimum value of inductance $\mathrm{L}$ and capacitance $\mathrm{C}$ ), which can discharge the required energy in specific pulse shape and duration, is essential for energizing the flash lamps. The flash lamp current pulse width and shape are controlled by two factors: (a) time constant $T$ (the rise time of the critically damped circuit) which is equal to $\sqrt{L C}$ and (b) damping constant $\alpha$, which is given as $\alpha=K_{0} / \sqrt{V_{0} Z_{0}}$, where $K_{0}$ is impedance constant for given flash lamp, $V_{0}$ is the charging voltage and $Z_{0}=\sqrt{L / C}$ is characteristic impedance of the circuit. The parameters $T$ and $\alpha$ are governed by the voltage and the capacitance at a given inductance of pulse-forming network. For delivering the same electrical energy to the flash lamp, higher capacitance at lower voltage increases the current pulse duration (which may result in loss of useful pump energy for $T \gg t_{f}, t_{f}=350 \mu \mathrm{s}$ ) whereas the smaller capacitance of circuit requires components of higher voltage ratings, such as relays, transformers, cables, etc. Thus causing increased cost of the PFN. Also the use of smaller capacitance for the PFN results in higher peak current in flash lamps, which would adversely affect the life of flash lamps.

Considering the above points, a dual polarity capacitor bank was designed and developed (Navathe et al 2001). It enables the operation of flash lamps at twice the rated capacitor voltage without need for using components of higher voltage ratings. In the case of xenon flash lamps used in the amplifier, the value of the parameter $K_{0}$ is calculated to be $50 \mathrm{VA}^{-0.5}$ and the value of the parameter $\alpha$ at $6 \mathrm{kV}$ is about $0 \cdot 78$. For the PFN with effective capacitance of $150 \mu \mathrm{F}$ and trigger transformer inductance of $70 \mu \mathrm{H}$, the circuit is critically damped and the value of $T$ is calculated to be $102 \mu \mathrm{s}$, which corresponds to total duration of current pulse (3T) equal to $306 \mu \mathrm{s}$.

Figure 4 depicts the circuit diagram of the power supply unit. There are two separate capacitor banks, one charged to + ve voltage and the other to -ve voltage. On the charging side, the line input of the three-phase mains transformer is connected through 3 solid-state relays (SSR). The transformer outputs are rectified and used to charge capacitor banks $\mathrm{C} 1$ to $+\mathrm{HV}$ and $\mathrm{C} 2$ to $-\mathrm{HV}$. The resistor divider networks $100 \mathrm{M} / 100 \mathrm{k}$ attenuate these voltages and feed them to the isolation amplifiers based on $\mathrm{V} / \mathrm{F}$ and $\mathrm{F} / \mathrm{V}$ converter and opto-coupler. This voltage is further given to inverting input of comparator (LM311) in the control module, which is part of the overall control system of the laser chain. The non-inverting input of the comparator is connected to a digital to analog converter, in which reference voltage is loaded by the control system. The comparator output in turn, is connected to SSRs through optocouplers. Thus, both the capacitor banks are charged to the set voltage. The triggering of the flash lamps is carried out with the help of trigger transformers, which receive external trigger pulses through the opto-coupler and trigger amplifier. The trigger transformer generates a pulse of $25 \mathrm{kV}$ with a pulse duration of few microseconds, which is sufficient to create ionization in the lamps, causing the main capacitor bank to discharge. The control circuit and the power supply are isolated from each other by an isolation amplifier and an opto-coupler.

The electronic control system of the laser is based on microprocessor (Navathe et al 2002; Gupta et al 1988). A control module is designed to control charging of capacitor bank in a typical amplifier and firing of the same in flash lamps at appropriate timing. This data is stored in the module by the microprocessor. The disc amplifier requires two control modules, one for positive bank and another one for negative bank.

The current pulse of the flash lamp discharge at a bank energy of $\sim 3.6 \mathrm{~kJ}$ (per flash lamp) using a current probe (LEM 6030/SD24) is shown in figure 5. The peak current through 


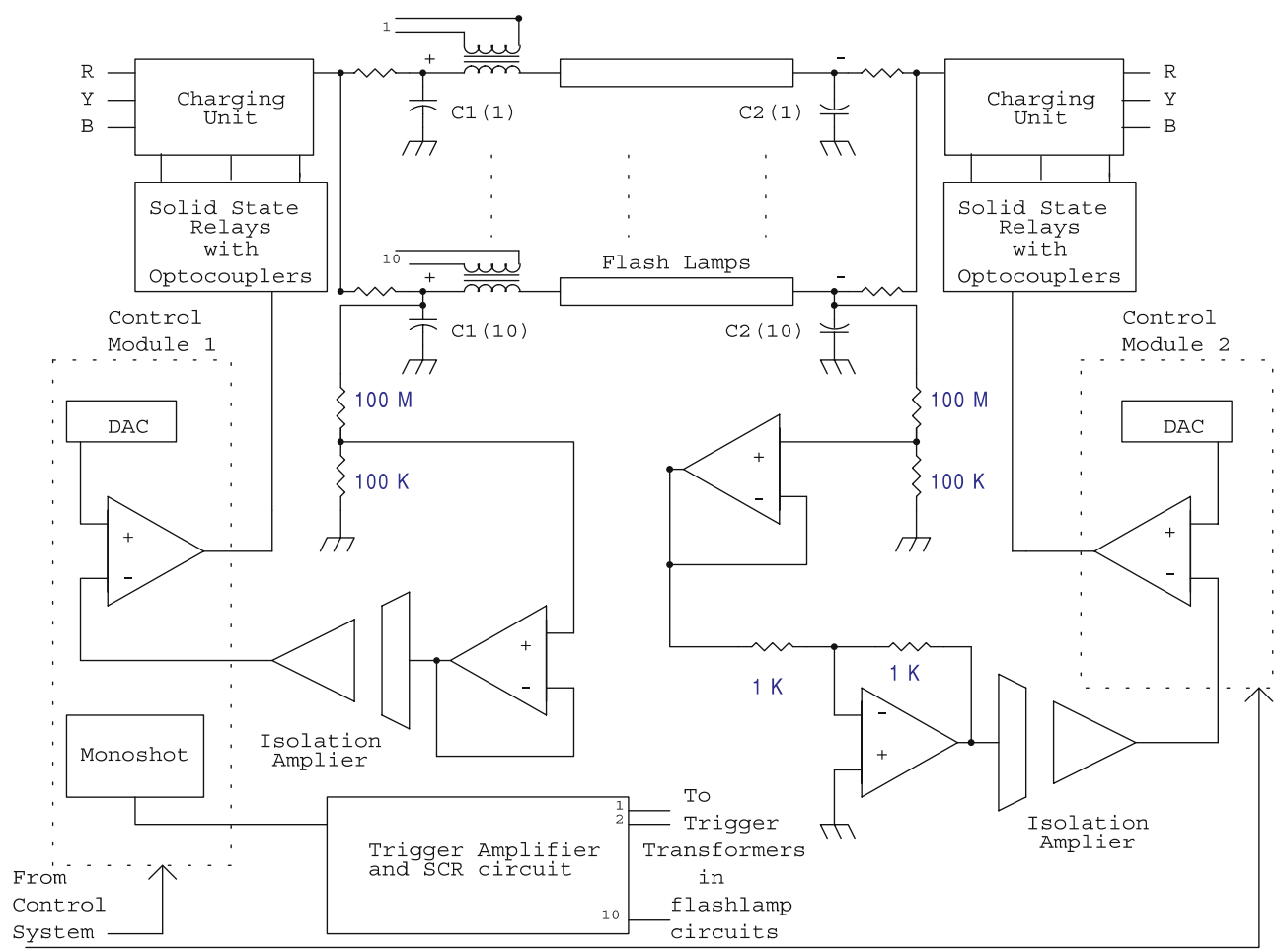

Figure 4. Schematic circuit diagram of bipolar power supply.

the flash lamp was found to be $4.4 \mathrm{kA}$ (current density of $1.7 \mathrm{kA} / \mathrm{cm}^{2}$ ), which is within the desired limits of current density for efficient pumping and long life. The current pulse width was measured to be $450 \mu \mathrm{s}$ (at $10 \%-10 \%$ of peak), which is larger than estimated value of $300 \mu \mathrm{s}$. This difference in the duration may be attributed to the higher inductance of the PFN due to non-saturation of the core of the transformer. It may be mentioned that the pulse width is greater than the upper level lifetime $\sim 320 \mu$ s of Nd in LHG-8 glass (Campbell \& Suratwala 2000).

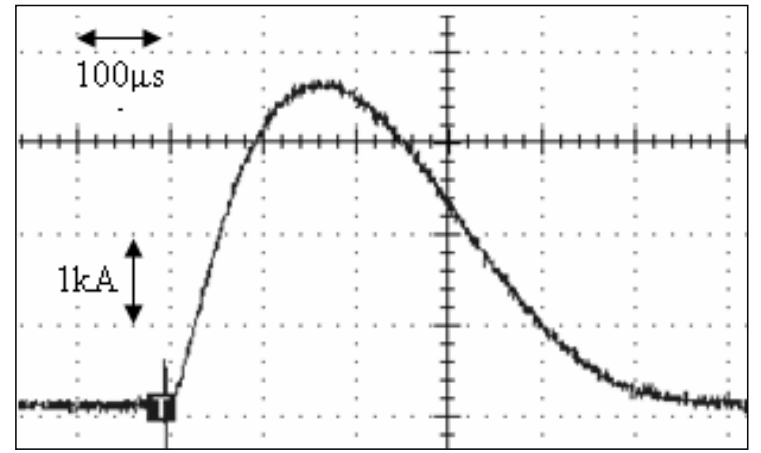

Figure 5. Waveform of the flash lamp current. 


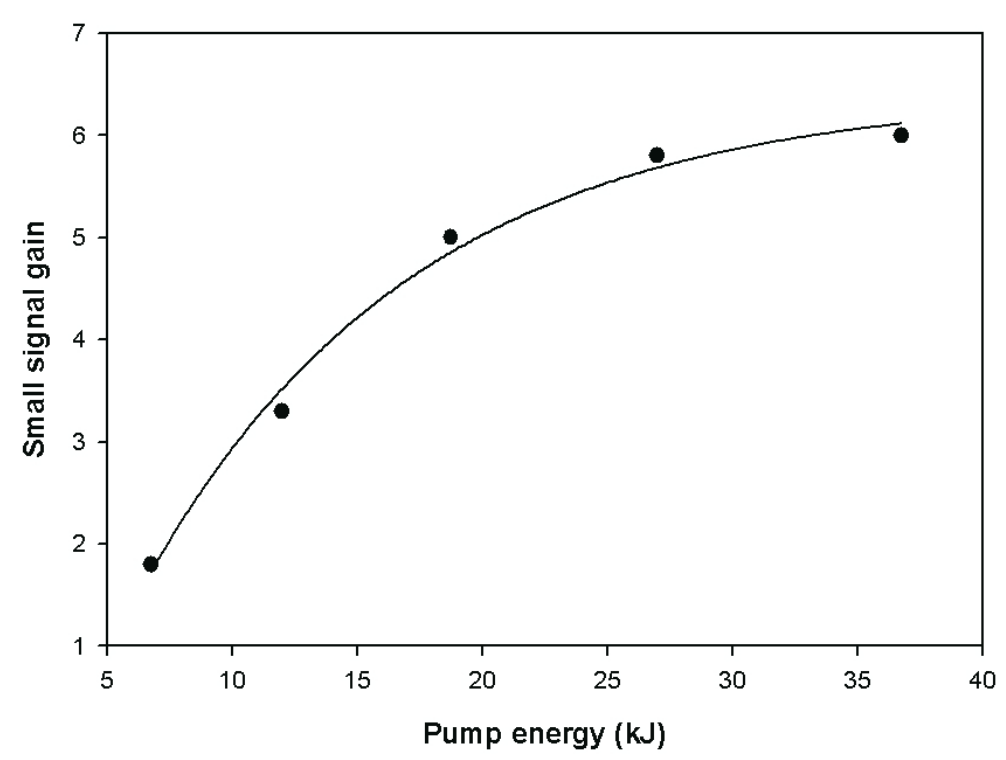

Figure 6. Small signal gain of the disc amplifier system as a function of input electrical energy.

\section{Performance characteristics}

Small signal gain of the disc amplifier was measured at the center of the laser discs as a function of the pump energy using a $10 \mathrm{~mJ}$ (15 ns FWHM) Q-switched Nd: YLF laser beam from one of the master oscillators of the high power laser chain. The pulse energy was measured at input and output of disc amplifier using pyroelectric energy meters. An interference filter having peak transmission at $1054 \mathrm{~nm}$ was used to avoid any contribution to the measured energy due to the flash lamp light. Due care was taken to minimize the contributions due to flash lamp emission at $1054 \mathrm{~nm}$ and ASE. A very small contribution to the measured signal arising due to these (as observed by blocking the input laser beam) was subtracted from the observed readings to calculate the gain. Figure 6 shows the single pass small signal gain as a function of the capacitor bank energy. It is observed from figure 6 , that the gain increases linearly with pump energy up to $\sim 20 \mathrm{~kJ}$, and thereafter saturates to a value $\sim 6$ at a pump energy of $36 \mathrm{~kJ}$. The stored energy was estimated to be $\sim 850 \mathrm{~J}$ at pump energy of $20 \mathrm{~kJ}$, from the measured small signal gain (using measured gain coefficient of $0.22 \mathrm{~cm}^{-1}$ for effective gain length of $7.2 \mathrm{~cm}$ ) and stimulated cross-section of $3.6 \times 10^{-20} \mathrm{~cm}^{2}$ (Campbell \& Suratwala 2000). The estimated stored energy matches well with the other reported values (Zhang et al 1998) for similar laser disc amplifiers.

The spatial gain variation in a disc may occur due to: (a) lower pumping of flash lamp energy in the edges, (b) due to excitation of parasitic oscillations because of poor quality cladding and (c) variation in flash lamp characteristics used for pumping. The variation in gain due to variations in currents of flash lamps was minimized using nearly identical flash lamps and the ring parasitic modes were suppressed using cladded laser discs. However, variation in gain mainly occurs due to poor pumping in peripheral region. The variation of gain as a function of spatial position across the cross-section of disc amplifier was measured using Nd: YLF master oscillator. Figure 7 depicts the single-pass, small-signal gain over beam cross-section of the disc amplifier. It is clear from this figure that gain decreases from $\sim 3 \cdot 1$ at center to 


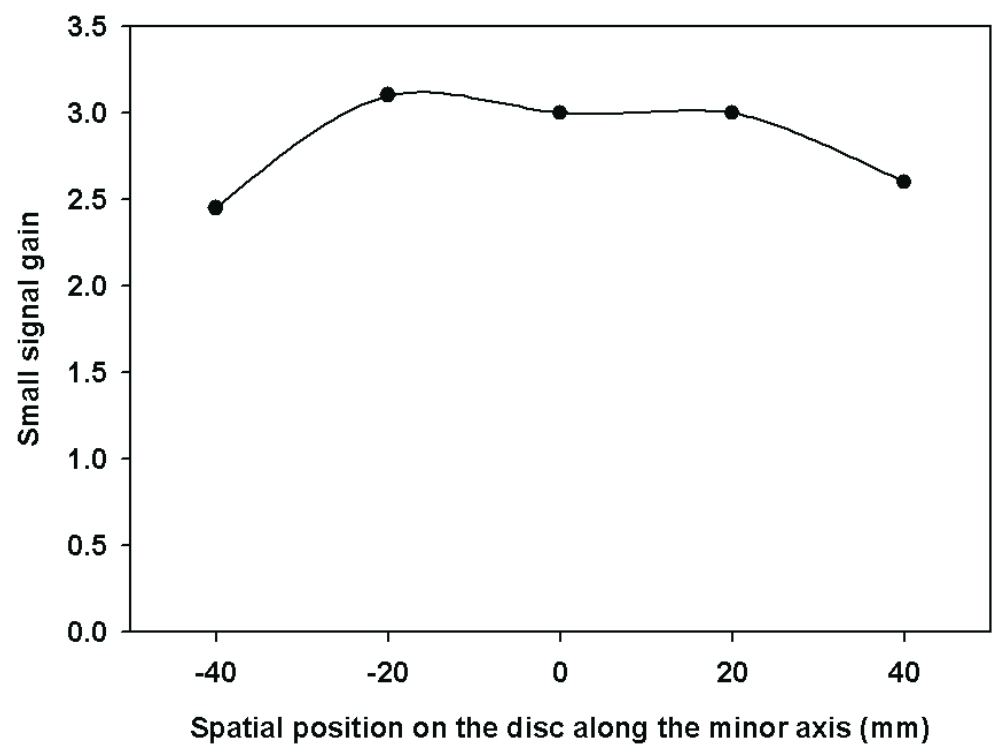

Figure 7. Spatial gain variation in the disc amplifier system.

$\sim 2.5$ at edges of the discs at a flash lamp pump energy of $10 \mathrm{~kJ}$. The gain was observed to be nearly constant over a beam cross-section of $\sim 50 \mathrm{~mm}$.

The observed peak of the fluorescence emission from the disc $(\sim 300 \mu \mathrm{s})$ with respect to the flash lamp trigger pulse matches fairly well with the reported values of upper level lifetime of $\mathrm{Nd}$ for $2 \cdot 2 \mathrm{wt} \%$ doped Nd in LHG- 8 phosphate glass. Hence it is seen that the loss due to ASE, parasitic oscillations and concentration quenching is minimum and it has not affected the fluorescence lifetime of the upper laser level of $\mathrm{Nd}$ in the present design of the disc amplifier. The ASE from the disc amplifier was observed to be $\sim 12 \mathrm{~mJ}$ at the pump energy of $36 \mathrm{~kJ}$.

The B-integral at an input intensity of $800 \mathrm{MW} / \mathrm{cm}^{2}$ and a small signal gain of 5 for the disc amplifier with glass thickness of $7.2 \mathrm{~cm}$ is $\sim \lambda / 20$, whereas it would be $2 \cdot 17 \lambda$ in the case of a rod amplifier of similar gain and length of $30 \mathrm{~cm}$.

The disc amplifier was then used as the last stage amplifier of one arm of the chain of twoarm Nd: phosphate glass laser system (figure 8). The laser beam from the $80 \mathrm{~mm}$ diameter amplifier having an energy of $\sim 50 \mathrm{~J}, 1.5 \mathrm{~ns}$ and diameter of $\sim 75 \mathrm{~mm}$ was coupled to the disc amplifier using a vacuum spatial filter (cut off frequency of 2.8 lines $/ \mathrm{mm}$ ) which had a magnification of 1.26 . The fill factor of the amplifier is $70 \%$. The pulse energy of $\sim 105 \mathrm{~J}$ was measured at the output of the disc amplifier. The stored energy of the disc amplifier matches well with the similar disc amplifier systems (Zhang et al 1998). The amplifier can be operated at a maximum fluence of $1.5 \mathrm{~J} / \mathrm{cm}^{2}$ for pulse duration of $1 \mathrm{~ns}$ mainly because of the LIDT of the optical components which is small compared to the saturation fluence $\sim 5.2 \mathrm{~J} / \mathrm{cm}^{2}$ for LHG -8 phosphate glass (Campbell \& Suratwala 2000). This restricts the operation of the disc amplifier to a wall plug extraction efficiency of $\sim 0.25 \%$ obtained at a bank energy of $20 \mathrm{~kJ}$.

The laser beam quality was tested using a shear interferometer. A set of straight-line parallel fringes were observed indicating that the laser beam is well collimated. Also, there was no 


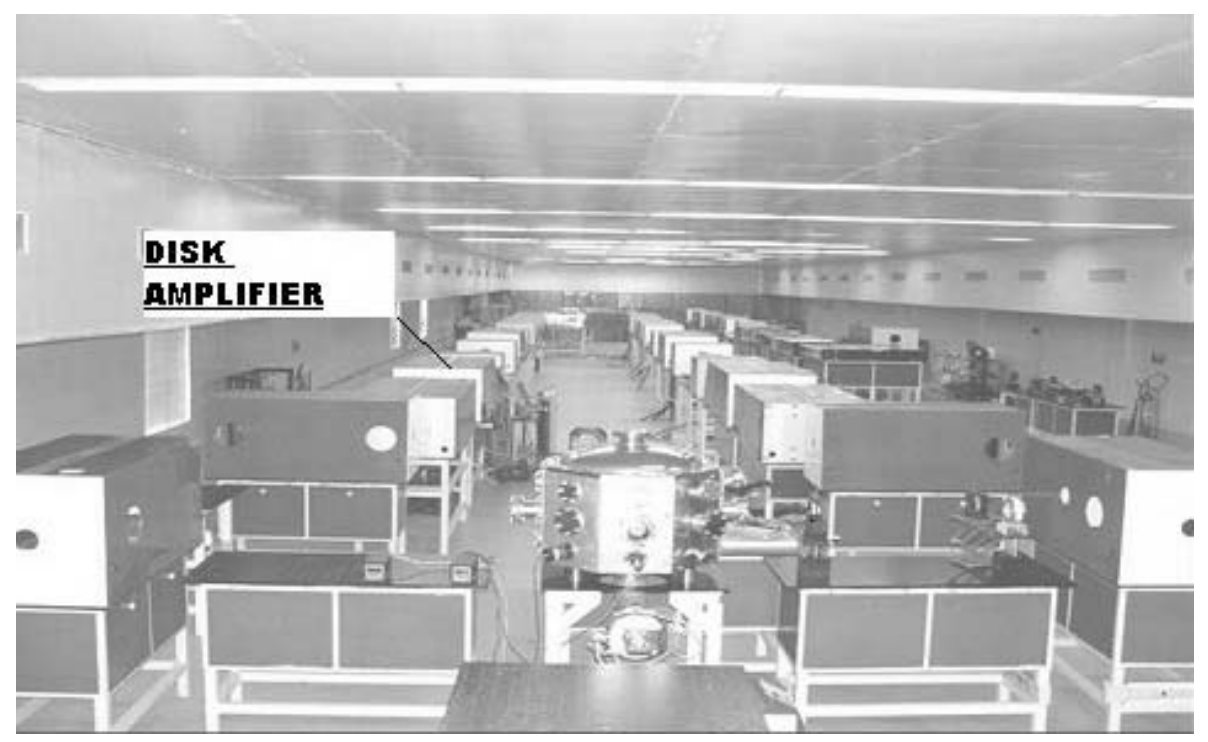

Figure 8. Photograph of the two-beam high power laser system.

detectable distortion of the fringes indicating that the aberrations are less than $\lambda / 4$ at the operating wavelength. The spectral width of the laser is $\sim 0.1 \mathrm{~nm}$. The polarization extinction ratio is $\sim 100: 1$.

\section{Conclusion}

A large aperture disc amplifier system has been designed, developed and characterized for its performance. Single-pass, small-signal gain of 6 at a pump energy of $36 \mathrm{~kJ}$ and its spatial variation have been experimentally measured. It uses an in-house designed and developed bipolar power supply, which delivers an electrical energy up to $40 \mathrm{~kJ}$ in pump pulse duration of $450 \mu$ s. It has been used as the last stage amplifier of high power Nd: glass laser system and provided output pulse energy in excess of $100 \mathrm{~J}$ at $1.5 \mathrm{~ns}$ pulse duration. The thermal recovery time of $\sim 20$ minutes has been measured.

The laser is being used for studies on generation of intense thermal $\mathrm{x}$-ray radiation in hohlraum cavities (Prasad et al 2003). By addition of the disc amplifier, the laser energy has increased which will further enhance the thermal $\mathrm{x}$-ray yield. This enhancement would make it possible to do experiments on $\mathrm{x}$-ray driven shocks in future.

\section{Acknowledgements}

Authors wish to thank the members of the High Power Laser Optics Laboratory and Laser Electronics Support Section for their technical help during the development of the amplifier. Thanks are also due to Dr S Chatterji for optical polishing of the laser discs. 


\section{References}

Bervekk R B 1975 EG\&G technical report-B-4426

Boehly T R, Brown D L, Craxton R S, Keck R L, Knauer J P, Kelly J H, Kessler T J, Kumpan S A, Loucks S J, Letzring S A, Marshall F J, McCory R L, Morse S F B, Seka W, Soures J M, Verdon C P 1987 Initial performance results of OMEGA laser system. Opt. Commn. 133: 495-506

Campbell E M, Hunt J T, Bliss E S, Speck D R, Drake R P 1986 NOVA experimental facility. Rev. Sci. Instrum. 57: 2101-2106

Campbell J H, Suratwala T I 2000 Nd-doped phosphate glasses for high-energy/high-peak-power lasers. J. Non-Cryst. Solids 263 and 264: 318-341

Erlandson A C, Rotter M D, Frank D N, McCarcken R W 1995 Design and performance of the beamlet amplifiers UCRL-LR-105821-95-1

Glaze J A, Guch S, Trenholme J B 1974 Parasitic suppression in large aperture Nd: Glass disc laser amplifiers. Appl. Opt. 13 12: 2808-2811

Gupta B L, Narayan B S, Navathe C P, Nalgundwar M, Bhawalkar D D 1988 Electronic control of high power Nd: Glass laser. BARC Internal Report \# I-937

Hunt J T, Glaze J A, Simmons W W, Renard P 1978 Suppression of self-focusing through low-pass spatial filtering and relay imaging. Appl. Opt. 17: 2053-2057

Joshi A S, Navathe C P, Ansari M S, Kamath M P, Bandhyopadhyay S, Upadhyay A, Kulkarni A P, Tripathi P K, Khare P, Patwa S R, Singh J D, Sreedhar N, Chandra R, Kumbhare M N, Pant H C 1999 Development of high power laser and relevant technology in India. Fusion Engineering and Design 44: 67-70

Koechner W 1988 Laser Amplifiers in Solid state laser engineering Second Edition, New York: Springer Verlag

Linford G J, Saroyan R A, Trenholme J B, Weber M J 1979 Measurements and modelling of gain coefficients for Neodymium laser glasses IEEE. J. Q. Electron 15: 6 510-523

Navathe C P, Ansari M S, Sreedhar N, Chandra R, Gupta S, Kulkarni A P, Kamath M P 2001 A bipolar power supply for Nd: Glass laser amplifiers, Proc. National Laser Symposium, CAT, Indore

Navathe C P, Ansari M S, Upadhayaya J, Nigam S, Sreedhar N 2002 A PC-based control system for multi-stage high power laser chain, Proc. National Laser Symposium, SCTIMST, Thirvanantpuram

Prasad Y B S R, Joshi A S, Senecha V K, Kamath M P, Khare P, Pant H C 2003 Proc. SPIE 5228: 263-273

Sutton S, Marshall C D, Petty C S, Smith L K, Van Wonterghem B M, Mills S 1998 Thermal recovery of NIF amplifiers SPIE 3492: 665-675

Touz'e Le G, Cabourdin O, Mengue J F, Guenet M, Grebot E, Seznec S E, Jancaitis K S, Marshall C D, Zapata L E, Erlandson A E 1998 3D gain modelling of LMJ and NIF amplifiers, Proc. of SPIE 3492: 630-637

Wonterghem B M V, Murray J R, Campbell J H, Speck R D, Barker C E, Smith I C, Browning D F, Behrendt W C 1997 Performance of prototype of a large-aperture multi-pass Nd: glass laser for inertial confinement fusion. Appl. Opt. 36 21: 4932-4953

Zhang W G, He S B, Zhang Xiao Min, Chunyu Y M, Li Y Z, Xue X G, Wei Xiaofeng, Man Y, Peng H 1998 Development progress for amplifier of SGIII laser facility Proc. of SPIE 3492: 586-591 